\title{
Erratum to: Standardisation and evaluation of a quantitative multiplex real-time PCR assay for the rapid identification of Streptococcus pneumoniae
}

Feroze A. Ganaie*, Vandana Govindan and K. L. Ravi Kumar

\section{Erratum}

In the publication of this article [1], there was an error in Table 1 which has a wrong sequence value at the GAPDH-probe.

The error:

'5' -Quasar 670-CTCAAGTTGGAAACCACGAGTAA GAGTGATGAA-3'-BHQ-2'.

Should instead read:

'5' -Quasar 670- CAAGCTTCCCGTTCTCAGCC-3' BHQ-2 :

This has now been included in this erratum.

Received: 26 May 2017 Accepted: 26 May 2017

Published online: 06 July 2017

\section{Reference}

1. Ganaie FA, Govindan V, Ravi Kumar KL. Standardisation and evaluation of a quantitative multiplex real-time PCR assay for the rapid identification of streptococcus pneumoniae. Pneumonia. 2015;6:1. doi:10.15172/pneu.2015.6/ 559.

* Correspondence: ferozeganaie@gmail.com

Hospital and Research Centre, Bangalore -560004, India 